\title{
Point of care testing: a welcome advance?
}

\author{
Peter T Scardino and Alexandra M Hay
}

Point of care (POC) testing refers to a laboratory assay that can be performed outside of a centralized facility, with results available to the physician and patient within minutes. One of the most common POC tests in medicine is the office urinalysis, performed by urologists for decades. The popularity of the home pregnancy test attests to the public's appetite for home testing. Now technology is available that will allow many clinical laboratory tests to be performed quickly, accurately and inexpensively by office staff or patients. Should urologists embrace POC testing for the office? If so, what problems might arise if patients can, for example, test their own prostate-specific antigen (PSA) levels at home, on demand?

The benefits of POC tests for some medical conditions have been well documented. Glycemic control in diabetic patients is better when blood glucose levels are measured regularly at the bedside or at home (Diabetes Control and Complications Trial Research Group [1993] N Engl J Med 329: 977-986). Control of anticoagulation is better when home testing of prothrombin time is used by the patient to adjust the dose of warfarin (Sawicki PT [1999] JAMA 281: 145-150). In each case, the benefits of POC testing arise from the availability of critical laboratory results in time for optimal medical decision making.

Despite the advantages, there are reasons to be concerned about POC testing. These tests are usually more expensive than when performed in a high volume centralized laboratory. Easy access to tests might lead to unnecessary utilization. By definition, the tests are carried out by medical staff or patients whose training, technical background and expertise in quality assurance are limited. POC testing may appear simple, but the instruments are complex and the potential for error is real.

Nevertheless, appropriate POC testing adds value to physician-patient encounters by introducing critical information during "...appropriate

POC testing

adds value

to physician-

patient

encounters

by introducing

critical

information

during the

consultation,

resulting

in better

decisions

made in less

time..."

PT Scardino is Editorin-Chief and AM Hay is Editor of Nature

Clinical Practice

Urology.

\section{Competing interests}

PT Scardino declared

an association with the

following company: Oxford

Bioscience. See the article

online for full details of

the relationship. AM Hay

declared no competing

interests.

www.nature.com/clinicalpractice doi: $10.1038 /$ ncpuro0878 the consultation, resulting in better decisions made in less time at no increased cost. Office visits and subsequent communications with the patient are often reduced when the key tests can be performed and the results received immediately.

The advantages of POC testing for PSA are evident - urologists could perform the test in their office, have the results available during the consultation in time for an immediate recommendation about further diagnostic testing or therapy; and the physician could profitably bill for the laboratory fee. If an office POC test for PSA became available, home testing would surely follow. In addition to home pregnancy tests, one can buy over-the-counter (OTC) tests for infertility, blood pressure and cholesterol. Recently a POC PSA test was described that measures levels between 0.5 and $25 \mu \mathrm{g} / \mathrm{l}$. (Karim O et al. [2007] Prostate Cancer and Prostatic Dis [doi: 10.1038/sj.pcan.4500962]). How would the public use such a test? The ability of a patient to interpret blood glucose levels is one thing, but interpreting a PSA results is more complex. PSA levels vary over time (Eastham JA et al. [2003] JAMA 289: 2695-2700) and must interpreted in the context of a patient's age, previous PSA results, and any prostatic manipulation (cystoscopy, biopsy) or disorders (infection, benign hypertrophy) or therapy (5- $\alpha$ reductase inhibitors, anticancer therapy). OTC PSA tests could lead to anxiety, even panic, as patients test their PSA frequently and seek advice on what the results mean. To counteract these effects would require an intense educational program.

The technology to provide POC testing for almost any laboratory test will soon be available. The benefits to urologists are so clear that POC testing for PSA is likely to become widespread. Surely it will not be long before such tests are made available to the public, for better or worse. 